\title{
The Effects of Stellar Yields with Rotation on Chemical Evolution Models
}

\author{
Leticia Carigi \\ Instituto de Astronomía UNAM, A.P. 70-264, DF 04510, Mexico
}

Max Pettini

Institute of Astronomy, Madingley Road, Cambridge CB3 OHA, England

\begin{abstract}
.
We summarise the results of recent work investigating the effects of stellar yields which take into account rotation on the predictions of chemical evolution models for early populations of galaxies.
\end{abstract}

As we have heard at this meeting, stellar rotation can affect significantly the chemical yields of stars over a wide range of stellar masses, from the most massive stars which drive powerful winds to the intermediate and low mass stars which evolve to the planetary nebula stage. We have begun to investigate some of these effects by constructing chemical evolution models for galaxies using rotation- and metallicity-dependent yields and comparing their predictions with those of models which do not include rotation in the adopted yields.

In particular we follow the detailed evolutions of the $\mathrm{C} / \mathrm{O}$ vs $\mathrm{O} / \mathrm{H}$ and $\mathrm{N} / \mathrm{O}$ vs $\mathrm{O} / \mathrm{H}$ ratios and compare them with available data for two populations of galaxies observed at high redshift $(z \simeq 3)$, when the universe (and presumably the galaxies) were young. The two populations are the Damped Ly $\alpha$ Systems (DLAs) seen in absorption in the spectra of bright background QSOs, and Lyman Break Galaxies (LBGs), star-forming galaxies recognised directly via their integrated stellar light.

Full description of the models is given in a forthcoming paper; here we summarise their principal features. LBGs and DLAs are treated as closed systems (i.e. no infall nor outflows are included in the models). In both cases we assume a total baryonic mass of $10^{10} \mathrm{M}_{\odot}$ with initial primordial composition. Their star formation rates $(\mathrm{SFR})$ are proportional to gas mass $\left(\mathrm{SFR}=\nu M_{\text {gas }}\right)$, where the efficiency $\nu$ is constant in time and depends on the type of galaxy. For LBGs we have assumed a high star formation efficiency with $\nu=1.0 \mathrm{Gyr}^{-1}$, while $\nu$ is ten times lower in DLAs. Qualitatively, this distinction is consistent with the lower luminosities and metallicities of DLAs, compared with LBGs at the same cosmic epochs (e.g. Pettini 2001).

We adopted the stellar yields by Meynet \& Maeder (2002) for stars of masses between 2 to $60 \mathrm{M}_{\odot}$ and for three values of metallicity, $Z=10^{-5}, 0.004$, and 0.02 (respectively $1 / 2000,1 / 5$ and 1 times solar metallicity). We interpolated linearly in metallicity and mass between the values published by Meynet \& Maeder (2002). The main results of our models are illustrated in Figure 1, and can be summarised as follows. 

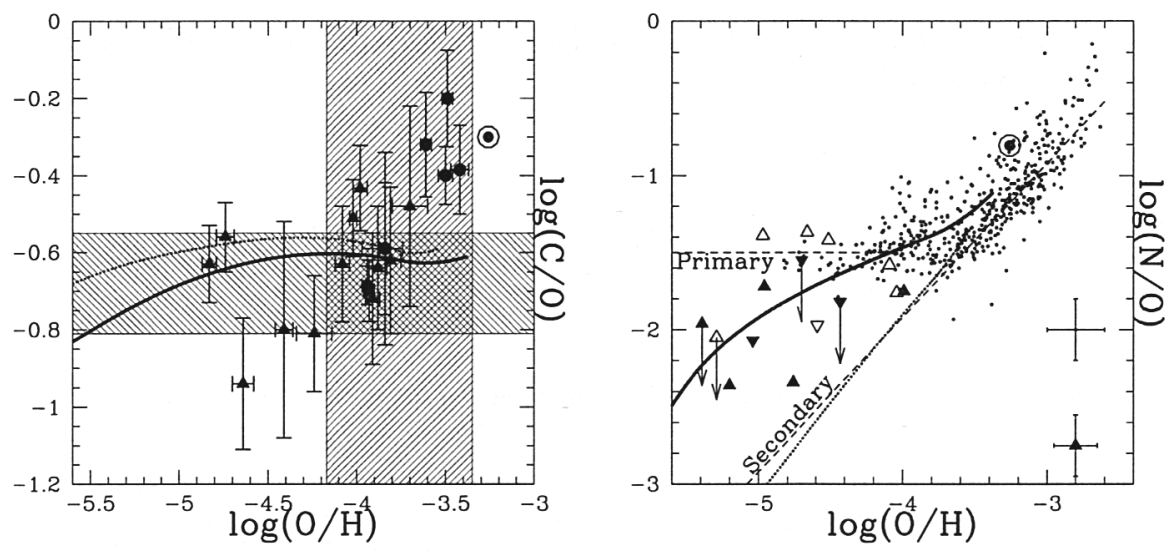

Figure 1. Left: $\log (\mathrm{C} / \mathrm{O})$ vs. $\log (\mathrm{O} / \mathrm{H})$ for LBGs and local HII regions (reproduced from Shapley et al. 2003, where full references to the data are given). Triangles are data from nearby dwarf Irregular galaxies and the Magellanic Clouds. Spiral galaxy data are shown with circles. The shaded areas indicate the likely ranges of values of $\log (\mathrm{C} / \mathrm{O})$ and $\log (\mathrm{O} / \mathrm{H})$ in LBGs. Right: Abundances of $\mathrm{N}$ and $\mathrm{O}$ in extragalactic $\mathrm{H}$ II regions (small dots) and damped Ly $\alpha$ systems (large triangles), reproduced from Pettini et al. (2002) (where full references to the data can be found). The dashed lines are approximate representations of the secondary and primary levels of $\mathrm{N}$ production. In both plots the solid and dotted curves show the predictions of our chemical evolution models using yields with and without rotation respectively. Solar abundances are indicated by the large bulls-eye.

1. Models with yields which take into account stellar rotation are successful in reproducing the $\mathrm{N} / \mathrm{O}$ ratios measured in DLAs (and local $\mathrm{H}$ II regions), whereas models without rotation do not match the data.

2. Our best fit to the DLA observations is obtained by adopting the yields with rotation and the initial mass function (IMF) by Kroupa, Tout \& Gilmore (1993). 3 . This same combination of yields and IMF can also reproduce the typical $\mathrm{C} / \mathrm{O}$ ratio recently estimated for LBGs. Although the $\mathrm{C} / \mathrm{O}$ ratio is apparently less sensitive to the effects of stellar rotation on the yields, models with rotation still seem to provide a better fit to the observations than those without.

\section{References}

Kroupa, P., Tout, C.A., Gilmore, G. 1993, MNRAS 262, 545

Meynet, G., Maeder, A. 2002, A\&A 390, 561

Pettini, M., 2001, in Gaseous Matter in Galaxies and Intergalactic Space, ed. R. Ferlet et al., (Frontier Group), 315

Pettini, M., Ellison, S.L., Bergeron, J., Petitjean, P. 2002, A\&A 391, 21

Shapley, A.E., Steidel, C.C., Pettini, M., Adelberger, K.L. 2003, ApJ 588,65 